7 INTRODUCTION OF MĀORI AND PACIFIC NURSE NAVIGATORS TO IMPROVE EQUITY IN SURGICAL PATHWAY IN AUCKAND, NEW ZEALAND

Andrew Jones, Dawson Ward, Pauline Fakalata. Auckland District Health Board, New Zealand

\subsection{6/bmjoq-2020-IHI.7}

Background Health outcomes for Māori and Pacific within New Zealand significantly poorer than for European New Zealanders. One identified inequity is the delay in receiving planned surgery. As a result of reduced surgery during the Covid-19 lockdown, these inequities were exacerbated.

Objectives Improve key metrics for Māori and Pacific patients receiving surgery (increase volume receiving surgery, decrease waiting times and decrease cancellation of surgery as well as identify systemic barriers to equitable care delivery.

Methods Ten Māori and ten Pacific nurse specialists were seconded to walk alongside patients awaiting surgery to identify and resolved immediate issues preventing progress. Care navigation by clinical staff, using Māori and Pacific health models in their interactions, allows patients to progress through their pathway through active engagement with the services. It is also allows for the gathering of data (quantitative and narrative) to understand where processes need to change to ensure equitable and timely care is provided. Through the experiences of those currently on the surgical wait list, alternative surgical models of care can be defined.

Results Early results suggest that the key metrics (days waiting, proportion of surgeries completed for Māori and Pacific patients and proportion of surgeries proceeding as planned) are improving. Qualitative feedback from patients and staff is clearly identifying opportunities as well as demonstrating the early value of having clinical staff supporting patients through their journey applying culturally safe and appropriate support.

Conclusions While still early in the development of the approach, initial results are highly promising and demonstrate that often what is missing in a patient's surgical journey is a consistent voice that provides certainty for patients and their families. Through the patient experiences gathered, service redesign will be possible that supports Māori and Pacific patients to achieve the outcomes they want.

\section{RAPID CYCLE IMPROVEMENT IN THE HOT ZONE}

Elizabeth Douglas, Kristina Fraser. University of North Texas Health Science Center, USA

\subsection{6/bmjoq-2020-|HI.8}

Background UNTHSC opened Fort Worth's first drive-through coronavirus testing site which functioned as both a community service and an opportunity for health professional students to learn rapid cycle improvement. Nasopharyngeal swabs were administered through vehicle windows in what was referred to as 'the hot zone.' Throughout 12 weeks many changes were made to the hot zone, including installing a tent, adding a second lane, improving hot zone staff training, and staggering opening times.

Objectives To ensure safe, efficient, and effective SARSCoV-2 testing for patients and staff.

Methods Total tests and times per test were recorded. Time in PPE was calculated from the time PPE was donned to average time doffed. Positive test count was used as a metric for pathogen exposure. Total cases among staff were also recorded along with near misses, incidents, and good catches as discussed in daily team debrief. Training was standardized and videos were created for hot zone and safety officer staff.

Results The site was open from March to June. With the tent, the accidental hot zone entry rate dropped from average of almost once per day to only once in 11 weeks. There were only 3 heat related incidents intercepted and 0 cases of COVID-19 among the 400 students and faculty. 8 safety incidents occurred. An average of 4 good catches or incidents prevented were counted per week. With staggering, time in full PPE was reduced from 165 minutes to 120 minutes. The

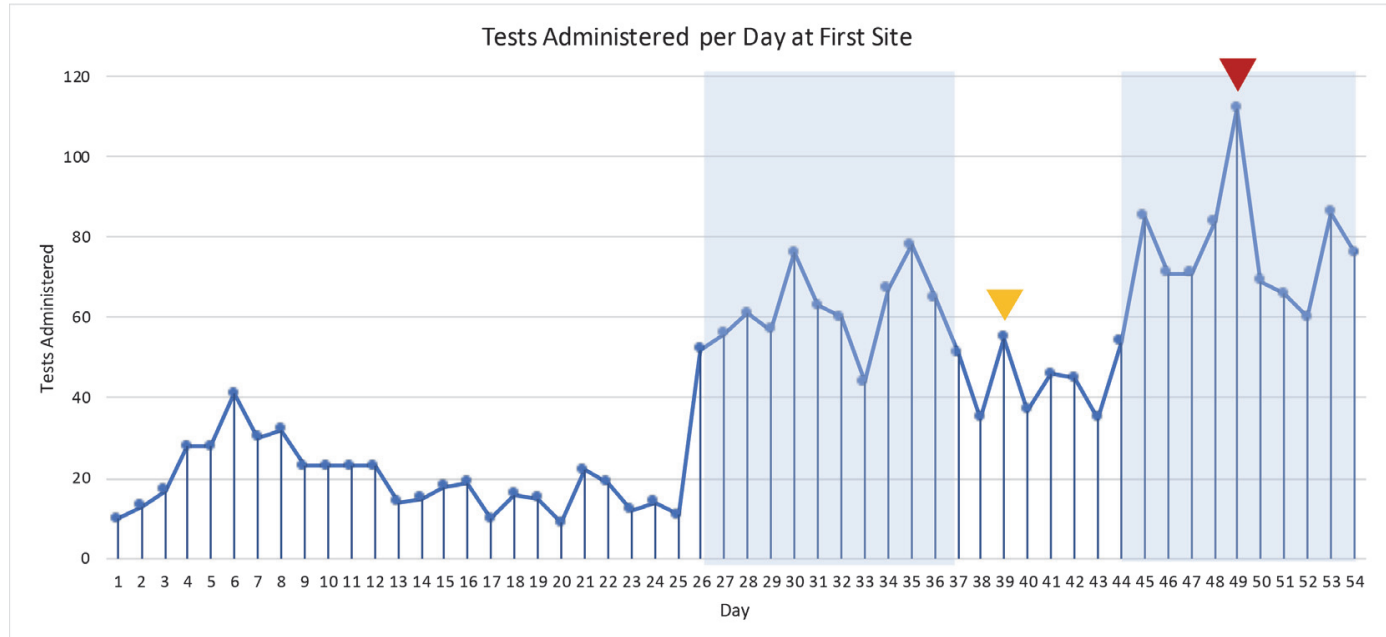

Shaded blue: 2 lanes per shift

Yellow triangle: 1 lane record

Red triangle: 2 lane record

Abstract 8 Figure 1 